\title{
Modeling Connectivity of Inter-Vehicle Communication Systems with Road-Side Stations
}

\author{
Wen-Long Jin ${ }^{*}$ and Hong-Jun Wang
}

Department of Automation, University of Science and Technology of China, P.R. China

\begin{abstract}
In this letter, we study the improvement that road-side stations can bring to the multihop connectivity of intervehicle communication in a traffic stream. With a recursive model of connectivity, we study impacts of different densities of road-side stations and different positions of road-side stations for uniform or non-uniform traffic streams.
\end{abstract}

Keywords: Vehicle infrastructure integration, inter-vehicle communication, connectivity.

\section{INTRODUCTION}

In recent years, more and more efforts have been devoted to applying wireless communication technologies to improve safety and operational efficiency of transportation systems $[1,2]$. Especially with the allocation of a block of spectrum from 5.850 to $5.925 \mathrm{GHz}$ for Dedicated Short Range Communications (DSRC) by US FCC, vehicle-to-vehicle (V2V) and vehicle-to-infrastructure (V2I) communications can be applied to relay various types of information. Information propagated through such road-side or on-board communication units would be useful for location- and time-critical applications. Such a vehicle infrastructure integration system can be naturally applied to relay traffic-related messages that are helpful for tackling transportation-related problems. For example, based on V2V and V2I communications, a so-called Autonet system is proposed to form an autonomous, self-organizing, transportation management, information, and control system [3]. Such a system has potential to redefine application domains of all existing intelligent transportation systems technologies [4], including Advanced Traffic Management Systems (ATMS), Advanced Traveler Information Systems (ATIS), Advanced Vehicle Control systems (AVCS), Commercial Vehicle Operations (CVO), Advanced Public Transportation Systems (APTS), and Advanced Rural Transportation Systems (ARTS). Therefore, to study and implement Vehicle Infrastructure Integration (VII) has been one of the major initiatives of the ITS Program in the US and other countries [5].

A conceptual VII system is illustrated in Fig. (1). In such a system, information can be exchanged among vehicles, traffic management centers, various elements of road infrastructure including traffic signals, message signs, bus stops, and other safety hardware. In particular, a DSRC enabled vehicle is capable of communicating with other vehicles and road-side stations. We hereafter refer to both V2V and V2I communications as IVC. That is, in an IVC system, a proportion of vehicles and all road-side stations are assumed to have communication capabilities. To improve performance of an IVC system, proper wireless communication technologies in both hardware and communication protocols have to be developed, appropriate transportation applications have to be identified, and the impacts of various traffic information on drivers'

*Address correspondence to this author at the Department of Automation, University of Science and Technology of China, P.R. China;

E-mail: wenlongjin@gmail.com various choice behaviors have to be understood. A fundamental problem of an IVC system is to understand the impact of road-side stations on its performance. This problem is related to practical decisions on where to install road-side stations to best improve the performance of the whole system. Among many performances of a wireless network is the multihop connectivity between two communication nodes. In an IVC system, the connectivity between two equipped vehicles or road-side stations is defined as the probability of existing a multi-hop communication path between them at a time instant [6].

In the literature, there are some studies on the impact of road-side stations on driving safety [7] and adaptive cruise control performance [8]. In [9], the impacts of the distribution of road-side stations on the connectivity of IVC system are studied with simulated data. In addition, there have been many studies on the connectivity of IVC system. In [10-12], vehicle movements are considered by using various traffic simulators. In [13], a new framework of instantaneous information propagation through IVC was proposed, and the assumption of instantaneous information propagation was supported by further analysis in $[14,15]$. In these studies, however, no road-side stations were included. That is, in literature, there lacks systematic approaches to modeling the multihop connectivity of an IVC system with road-side stations. In this paper, we attempt to develop a model for evaluating impacts of road-side stations on connectivity of an IVC system with similar conceptual framework of [13]. In this model, in addition to the distribution pattern of vehicles, market penetration rate of wireless communication units in vehicles, and transmission range, the distribution pattern of road-side stations would significantly affect the performance in connectivity of the IVC system.

The rest of the article is organized as follows. In Section 2, we introduce an analytical model of an IVC system with roadside stations. In Section 3, we analyze the connectivity of an IVC system under different conditions. In Section 4, we summarize our findings and present some discussions.

\section{AN ANALYTICAL MODEL OF CONNECTIVITY OF IVC SYSTEMS WITH ROAD-SIDE STATIONS}

\subsection{Conceptual Framework}

As shown in Fig. (2), we consider a stream of vehicles on a road link, besides which are some road-side stations. In this 


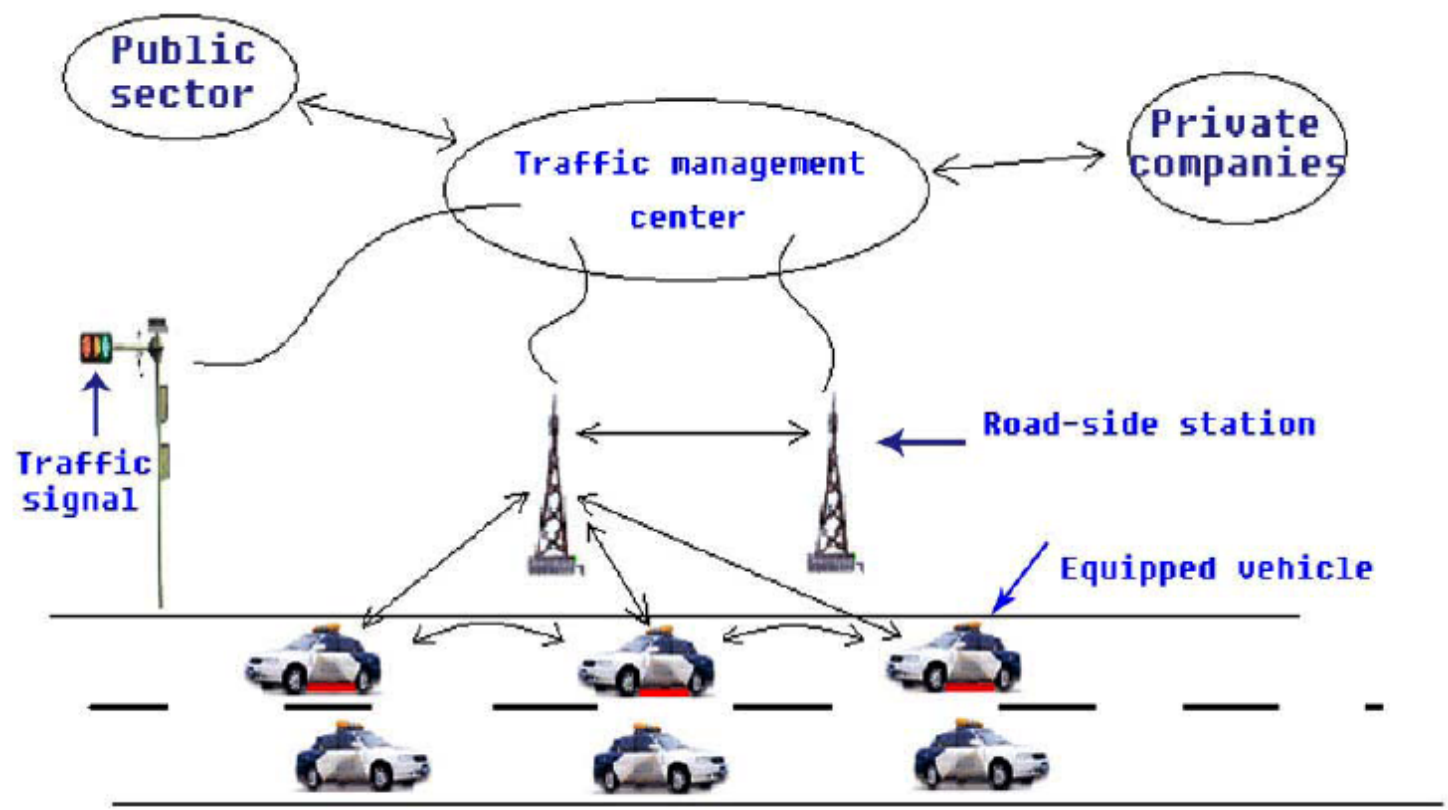

Fig. (1). An illustration of conceptual vehicle infrastructure integration systems.

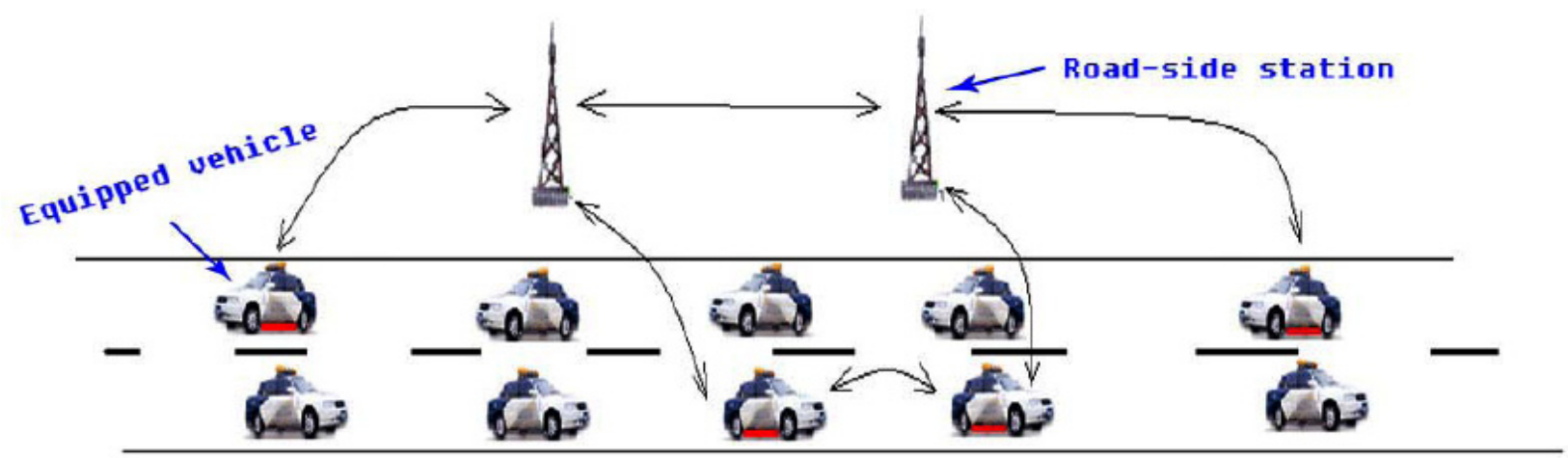

Fig. (2). An inter-vehicle communication system with road-side stations.

system, all road-side stations are capable of communicating. In the traffic stream, the locations of all vehicles are predetermined from observations or simulations, but only a portion of vehicles are equipped with wireless communication units. We assume whether a vehicle is equipped or not follows a uniform distribution, and the probability of each vehicle to be equipped is $\mu \in(0,1]$. The transmission range of a wireless communication unit is $R$. Further, in the traffic stream, the first and last vehicles are assumed to be equipped with wireless communication units. In this study we attempt to calculate the probability for the two vehicles to be connected through IVC. This is equivalent to studying the probability for all equipped vehicles in the whole traffic stream to be connected.

To formulate the problem mathematically, we introduce the following conceptual framework. Since such an IVC system is symmetric for two directions of information propagation, we only consider the positive direction of the $x$-axis. Further, we assume there are $S$ road-side stations along the traffic stream. We denote the connectivity between the first and last equipped vehicles of the traffic stream as $\tau$, which is also called the connectivity of the whole traffic stream. We can divide the whole traffic stream into $S+1$ sub-streams at road-side stations. In sub-stream $S$ $(s=0, \cdots, S)$, we label vehicles as $(s, i)$, where $i=0, \cdots, K_{s}$ increases in the direction of $x$-axis. In this notation system, the first vehicle in the traffic stream is $(0,0)$, road-side station $s(s=1, \cdots, S)$ is $\left(s-1, K_{s-1}\right)$ and $(s, 0)$ since it is both the end of sub-stream $s-1$ and the start of sub-stream $s$, and the last vehicle in the traffic stream is $\left(S, K_{S}\right)$. Here $K_{s}+1(s=0, \cdots, S)$ is the number of vehicles in sub-stream $s$.

As we know, $R, \mu$, distribution of vehicles in a traffic stream, and distribution of road-side stations can all affect the connectivity of the traffic stream, $\tau$. In the following, we will develop a model of the connectivity with these parameters.

\subsection{An Analytical Model of Connectivity}

We first study the connectivity of sub-stream $s, \tau_{s}$, which equals the connectivity between $(s, 0)$ and $\left(s, K_{s}\right)$ for $s=0, \cdots, S$. Since there are no road-side stations within a sub-stream, the problem becomes the same as that studied in $[14,15]$. We can obtain the connectivity $\tau_{s}$ with the analyti- 
cal model in [14] or the Monte Carlo simulation model in [15].

Here we introduce an analytical model for computing the connectivity of a sub-stream with vehicles 0 to $K$. We first split the sub-stream into cells as follows: All vehicles within the transmission range of, but not including, the information source, i.e. vehicle 0 , belong to cell 1 ; all vehicles within the transmission range of, but not including, any vehicle in cell $c$ belong to cell $c+1$. Without loss of generality, we only consider the communication manner of "most forwarded within range" [14]. That is, we denote information source as node 0 , and the farthest vehicle that node $h$ can communicate with in a hop is node $h+1$. Here $k^{*}$ denotes the farthest downstream vehicle within the transmission range of vehicle $k$, and $k_{*}$ the farthest upstream vehicle. Then the node probability, i.e. the probability for vehicle $k$ to be node $h$, and the end node probability, i.e. the probability for vehicle $k$ to be node $h$ and node $h$ to be the last node in a communication chain, are denoted by $P(k ; h)$ and $\bar{P}(k ; h)$, respectively. In addition, we denote $Q(j, k ; h)$ as the probability of the joint event of $(j ; h-1)$, for vehicle $j$ to be node $h-1$, and $(k ; h)$, for vehicle $k$ to be node $h$. That is, $Q(j, k ; h)$ is the probability for a hop from node $(j ; h-1)$ to node $(k ; h)$. Based on the analysis of the regulatory properties of the so-called most-forwarded-within-range communication chains spanning over cells, regressive models of node or end node probabilities can be written as follows [14]. First, we have $P(0 ; 0)=1$, since the information source is always node 0 in our model. Since node $h$ has to be the end of a hop from node $h-1$ to node $h$, we have the following relationship between node and hop probabilities

$$
P(k ; h)=\sum_{j=k_{*}}^{k-1} Q(j, k ; h)
$$

Further, hop probability can be computed recursively as follows:

$$
Q(k, l ; h+1)=\left(P(k ; h)-\sum_{j=l_{*}}^{k-1} Q(j, k ; h)\right) \mu v^{k^{*}-l}
$$

where $v=1-\mu$. Then node $h$ is the end node if it cannot be the start of a hop to node $h+1$. Thus end node probabilities can be written as

$$
\bar{P}(k ; h)=P(k ; h)-\sum_{i=k+1}^{k^{*}} Q(k, i ; h+1)
$$

Further, the probability for vehicle $k$ to an end node regardless of the number of hops is defined as $\bar{P}(k)=\sum_{h} \bar{P}(k ; h)$, the probability for information to travel to or beyond vehicle $k$ is $s(k)=\sum_{i \geq k} \bar{P}(i)$, and the connectivity of the sub-stream can be obtained as

$$
\tau(K)=s\left(K_{*}\right)
$$

since vehicles 0 and $K$ are connected if and only if information can travel to or beyond the farthest upstream vehicle of
$K$, i.e., vehicle $K_{*}$. The analytical model has been crossvalidated with a Monte Carlo simulation model developed in [15]. In these studies, however, no road-side stations have been incorporated in the model. In this paper, we attempt to extend the model for the scenario when road-side stations are placed along a traffic stream with equipped vehicles.

In an IVC system, road-side stations could serve as gateways for vehicles to communicate with transportation authorities or connect to a broader network such as internet. In addition, road-side stations would be critical to encourage the adoption of IVC by individual drivers or car companies, since it is expected to take years to have a market penetration rate so that $\mathrm{V} 2 \mathrm{~V}$ communications become useful even when IVC has been shown to be feasible with current communication technologies [3]. That is, road-side stations could help to extend the communication distance and improve multihop connectivity of IVC.

Here we propose a model of multihop connectivity of IVC in a traffic stream and attempt to quantify the benefit of introducing road-side stations along the traffic stream. First, we divide the traffic stream into more than one sub-streams at road-side stations. Then we can observe the following property on its connectivity: The whole traffic stream is connected if and only if all the sub-streams are connected. That is,

$$
\tau=\tau_{0} \tau_{1} \cdots \tau_{S}
$$

for a traffic stream with $S$ sub-streams. This property can be explained as follows. If the first and last vehicles are connected, there exists at least one communication path between them. Then any road-side station between them is either a node of the communication path or not. For a road-side station not being a node of the communication path, there must exist a hop connecting two nodes on its two sides, and we can break the hop into two with the road-side station as an extra node. That is, all sub-streams are connected. On the other hand, if all sub-streams are connected, we can obtain a communication path between the first and last vehicles by connecting all communication paths in the sub-streams. In this sense, the connectivity of the whole traffic stream is the joint probability for all sub-streams to be connected; i.e., $\tau=\tau_{0} \tau_{1} \cdots \tau_{S}$.

In Fig. (3), we summarize the algorithm for computing connectivity of IVC in a traffic stream with road-side stations. In this algorithm, we first divide the traffic stream into a number of sub-streams at road-side stations, then compute the connectivity of each sub-stream, and finally compute the connectivity of the whole traffic stream with Equation 5.

\section{NUMERICAL RESULTS}

In this section, we study the connectivity of an IVC system with road-side stations under various conditions. In these studies, we assume that $10 \%$ of all vehicles are equipped with wireless communication units; i.e., $\mu=0.1$. The transmission range of all equipped vehicles and roadside stations is $200 \mathrm{~m}$.

\subsection{Impact of Densities of Road-Side Stations}

We consider a uniform traffic stream with traffic density of $180 \mathrm{veh} / \mathrm{km}$ and length of $10 \mathrm{~km}$. In Fig. (4), the solid line 


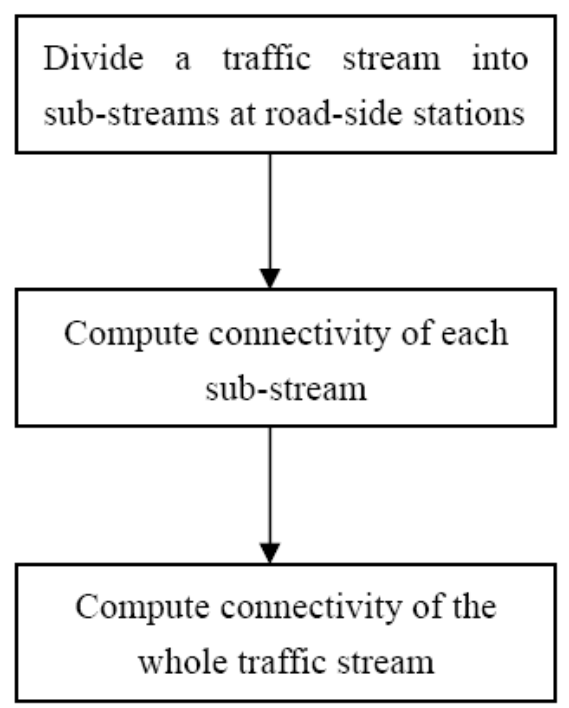

Fig. (3). A flow-chart of the algorithm for computing connectivity of an IVC system with road-side stations.

demonstrates the connectivity between two equipped vehicles at different distances without any road-side stations installed. The dashed, dotted, and dash-dotted lines show the connectivity when nine, 19 , and 39 road-side stations are evenly distributed along the traffic stream, respectively. That is, in the latter three scenarios, two consecutive road-side stations are $\mathrm{D}=1000,500$, and $250 \mathrm{~m}$ apart, respectively. From this figure, we can see that road-side stations can improve connectivity of a traffic stream and more road-side stations yield better performance. From the solid curve for the case without road-side station along the traffic stream, we can observe a discontinuity at $200 \mathrm{~m}$ : for vehicles within the transmission range of the information source, their connectivity is perfect 1 ; for a vehicle just outside of the transmission range, its connectivity is $1-v^{36}$, which is the probability when none of the 36 vehicles within the transmission range is equipped with a wireless communication unit. Similarly, the other curves have a shape of staircase, since all vehicles within the downstream transmission range of a road-side station share the same connectivity to the information source. Note that, if we place road-side stations every $200 \mathrm{~m}$ or less apart, we expect to have perfect connectivity along the traffic stream.

\subsection{Impact of positions of Road-Side Stations}

We first consider a uniform traffic stream from $\mathrm{x}=0$ to $\mathrm{x}=2000 \mathrm{~m}$ with density of $180 \mathrm{veh} / \mathrm{km}$. Here we only install one road-side station along the traffic stream. Fig. (5) shows the connectivity of the traffic stream for different positions of the road-side station. From the figure, we can see that, as expected, there is no improvement in connectivity when the road-side station is too close to two end points. The discontinuity in the connectivity can be explained similarly as in the preceding subsection. However, it is quite counter-intuitive that there are two best positions for the road-side station that are on the verge of the transmission range $(200 \mathrm{~m})$ of the first and last vehicles. Since the problem is totally symmetric, we expected the best position to install the road-side station would be the middle point.

Due to the existence of various bottlenecks in a road network, such as lane-drops, merging and diverging junc-

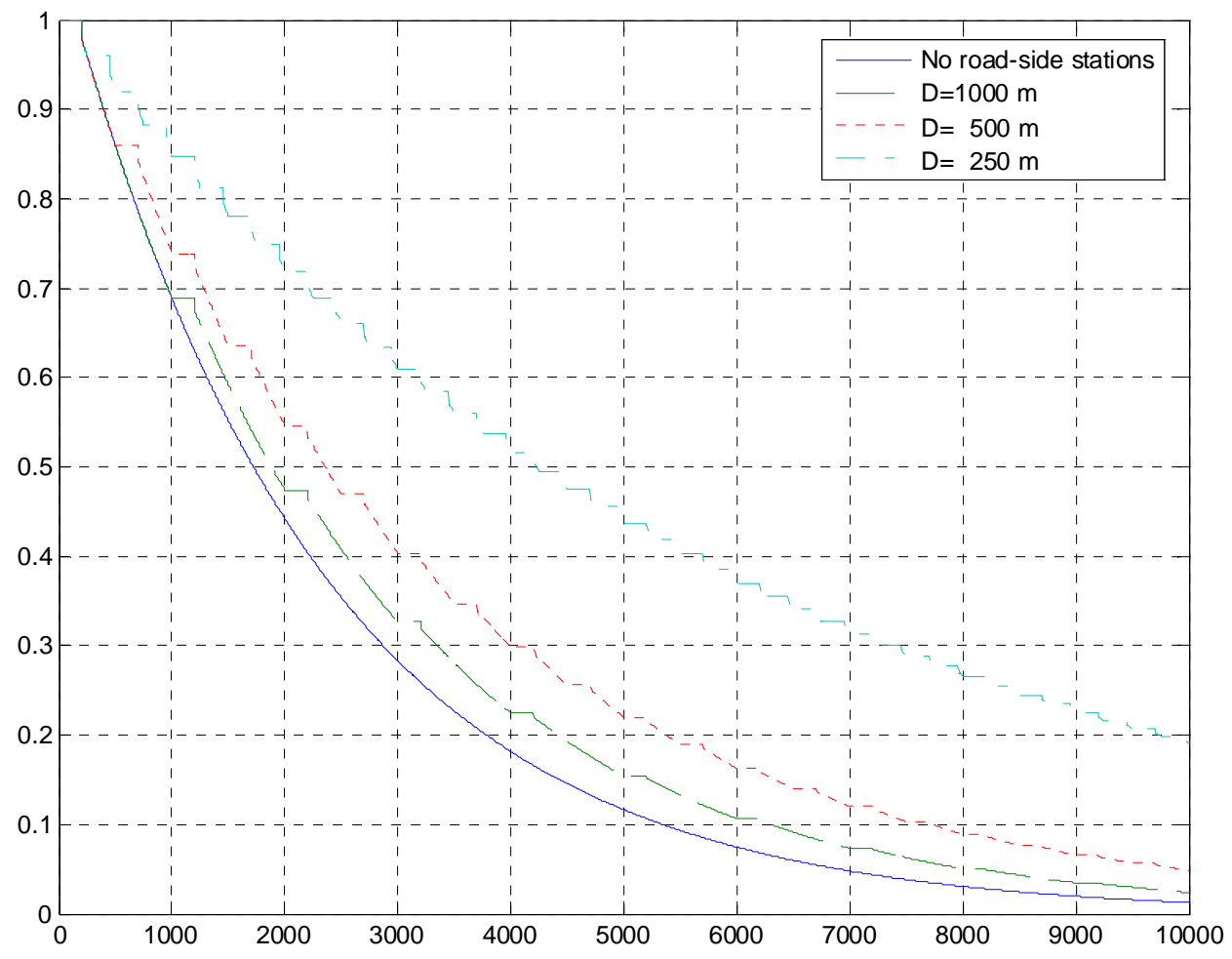

Fig. (4). Impacts of densities of road-side stations on connectivity. 


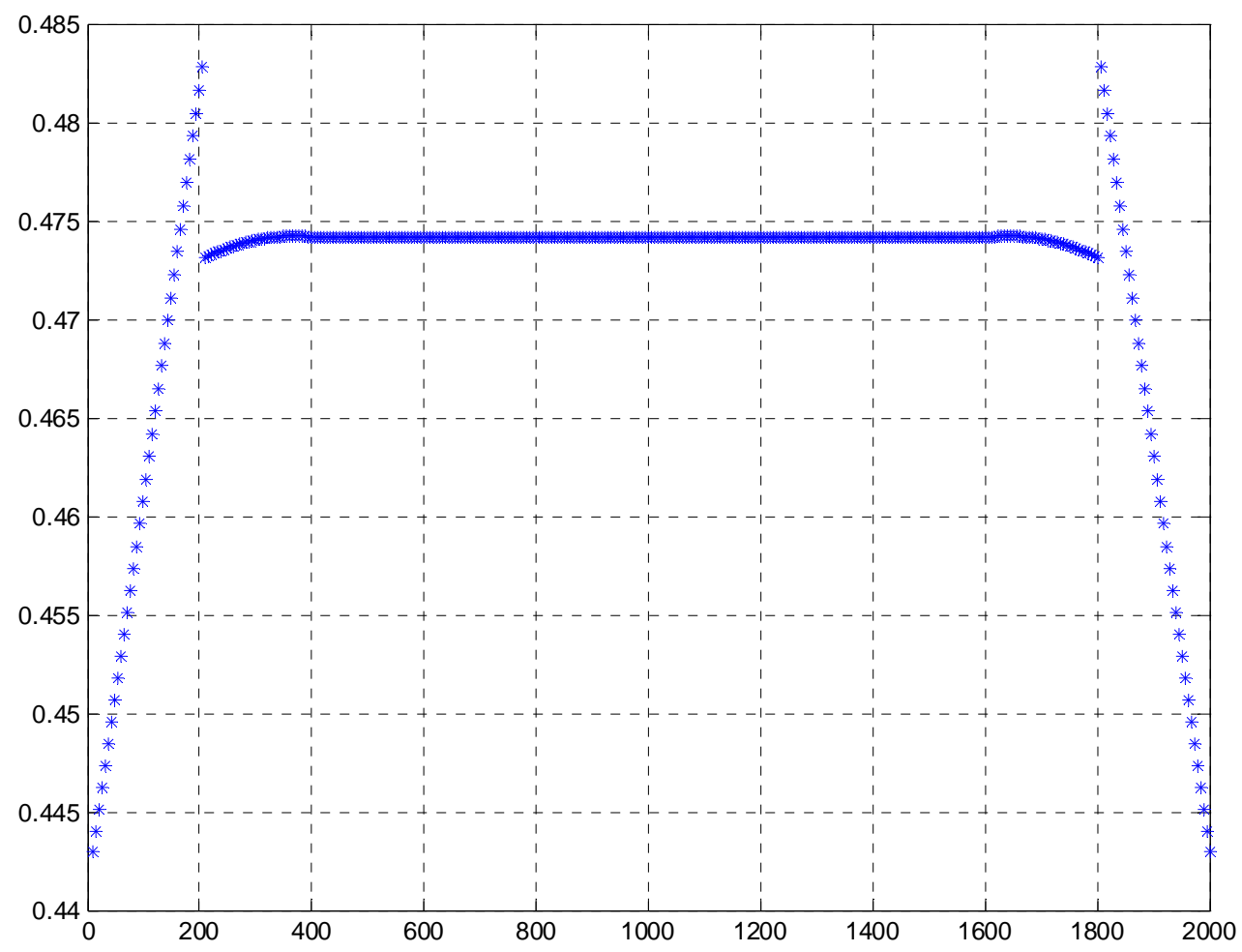

Fig. (5). Connectivity of a uniform traffic stream for different positions of a road-side station.

tions, traffic signals, traffic conditions are generally not uniform. Therefore, it is important to consider the impact of road-side stations on the multihop connectivity for vehicles in non-uniform traffic. Since the model proposed in section 2 is applicable for any distribution patterns of vehicles, we are able to study the impact of road-side stations for any traffic scenarios. As an example, here we consider a piecewise uniform traffic stream from $\mathrm{x}=0$ to $\mathrm{x}=2000 \mathrm{~m}$ with density of $180 \mathrm{veh} / \mathrm{km}$ for the first quarter, $100 \mathrm{veh} / \mathrm{km}$ for the second, $200 \mathrm{veh} / \mathrm{km}$ for the third, and $150 \mathrm{veh} / \mathrm{km}$ for the fourth. Fig. (6) demonstrates connectivity of the traffic stream when installing only one road-side station at different locations along the traffic stream. From the figure, we can see that better improvement in connectivity is obtained when the road-side station is installed along the sparser traffic.

\section{CONCLUSION}

In this paper, we first presented an analytical model for the multihop connectivity of an IVC system formed by a stream of vehicles and road-side stations. We then studied the impacts of road-side stations on the multihop connectivity for different distribution patterns of road-side stations and different traffic conditions. From the numerical studies, we find that, as expected, road-side stations can help relay information in an IVC system and have the following impacts on the performance of an IVC system:

(1) The larger the density of road-side stations, the more improvement in the connectivity.

Different locations of road-side stations relative to a traffic stream can yield different levels of improve- ment in connectivity. In particular, it is better to deploy road-side stations along less congested traffic. This is consistent with the observation that sparse traffic is the communication bottleneck of an IVC system. For a particular road network, the best locations of road-side stations can be calculated with the proposed model.

In addition to considerations in performance, economic constraints should also be considered when deploying roadside stations, since available budget would limit the number of road-side stations and may yield sub-optimal results. In this case, the analytical model is still helpful for estimating IVC performance for different economic scenarios. In this sense, the proposed model can be used to design a system with an economically reasonable number of road-side stations and with acceptable performance. Due to its high computational efficiency compared with simulation-based approaches, this model would be especially helpful, should some emergency vehicles with wireless communication capabilities be deployed in field to resume or improve intervehicle communication when disasters like earthquakes knock off other communication systems.

In the future, we will look into deployment strategies of road-side stations for a road network and how road-side stations would affect performances of IVC in terms of communication throughput, communication network stability, etc. We will also be interested in how road-side stations would affect drivers' driving and route-choice behaviors. 


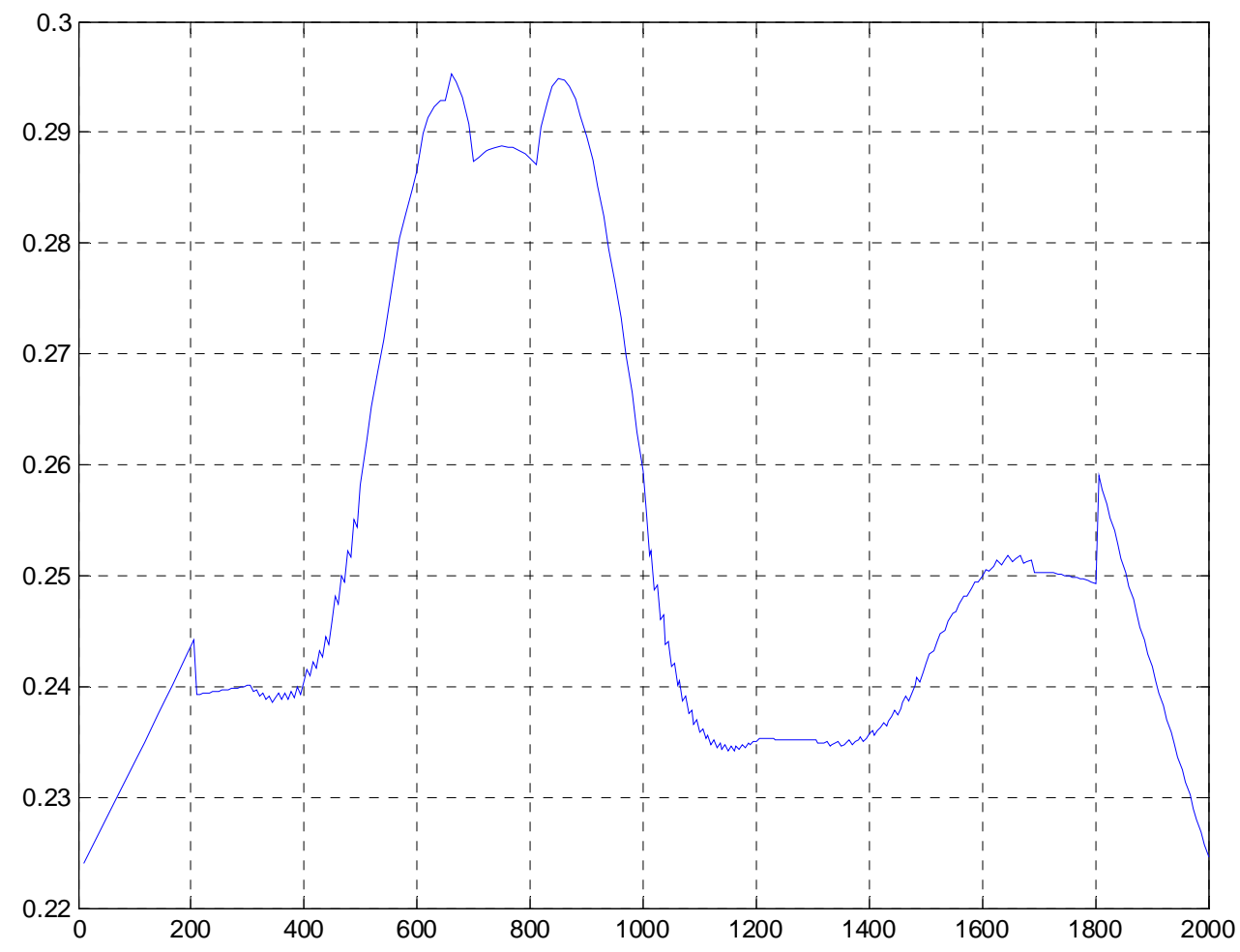

Fig. (6). Connectivity of a piecewise uniform traffic stream for different positions of a road-side station.

\section{ACKNOWLEDGEMENTS}

This work was supported in part by National Natural Science Foundation of China (No. 50708107), Hi-Tech Research and Development Program of China (863 Project) (No. 2007AA11Z222), and the Science Research Fund of MOE-Microsoft Key Laboratory of Multimedia Computing and Communication (Grant No. 06120810). The views and results contained herein are the authors' alone and do not necessarily reflect those of the sponsors.

\section{REFERENCES}

[1] J. Hedrick, M. Tomizuka, and P. Varaiya, "Control issues in automated highway system", IEEE Control Syst Mag N. Y., vol. 14(6), pp. 21-32, 1994.

[2] http://www.cartalk2000.net/. Accessed November 11, 2003.

[3] W. W. Recker, W.-L. Jin, X. Yang, and J. Marca, "Autonet: Intervehicle communication and network vehicular traffic", International Journal of Vehicle Information and Communication Systems, 2007. Forthcoming.

[4] J. M. Sussman, Perspectives on intelligent transportation systems, Springer Science and Business Media: New York, 2005.

[5] USDOT'S ITS Program - Major Initiatives - 2004. http://www.its. dot.gov/press/Initiatives4.htm.Accessed January 30, 2007.

[6] L. Briesemeister, L. Schafers, and G. Hommel, "Disseminating messages among highly mobile hosts based on inter-vehicle communication", In IEEE Intelligent Vehicles Symposium, pp. 522-27, OCT 2000 .
[7] W. Chen and S. Cai, "Ad hoc peer-to-peer network architecture for vehicle safety communications", IEEE Communications Magazine., vol. 43(4), pp. 100-7, 2005

[8] Q. Xu, K. Hedrick, R. Sengupta, and J. VanderWerf. "Effects of vehicle-vehicle/roadside-vehicle communication on adaptive cruise controlled highway systems", In Proceedings of Vehicular Technology Conference, 2002.

[9] J. Crabtree and N. Stamatiadis, "Using dedicated short-range communications technology for freeway incident detection: Performance assessment based on traffic simulation data", In Proceedings of Transportation Research Board Annual Meeting, 2007.

[10] H. Hartenstein, B. Bochow, A. Ebner, M. Lott, M. Radimirsch, and D. Vollmer, "Position-aware ad hoc wireless networks for intervehicle communications: the fleetnet project", In Proceedings of the 2nd ACM international symposium on Mobile ad hoc networking \& computing, pp. 259 - 62, Long Beach, CA, USA, 2001.

[11] H. Wu, R. Fujimoto, and G. Riley, "Analytical models for information propagation in vehicle-to-vehicle networks", Vehicular Technology Conference, vol. 6, pp. 4548 - 52, 2004.

[12] X. Yang and W. W. Recker, "Simulation studies of information propagation in a self-organizing distributed traffic information system", Transportation Research Part C, vol. 13(5-6), pp. 370-90, 2005.

[13] W.-L. Jin and W. W. Recker, "Instantaneous information propagation in a traffic stream through inter-vehicle communication", Transportation Research Part B: Methodological, vol. 40(3), pp. 230-50, March 2006.

[14] W. -L. Jin and W. W. Recker, "An analytical model of multihop connectivity of inter-vehicle communication systems". In revision, 2006.

[15] W.-L. Jin and W. W. Recker. "A monte carlo simulation model of inter-vehicle communication", Transportaton Research Record: Journal of Transportation Research Board, 2000: 8-17, 2007. 